SEÇÃO: ARTIGOS

\title{
ATIVIDADES PRÁTICAS EM CENTROS DE ATENÇÃO PSICOSSOCIAL COMO ESTRATÉGIA NA FORMAÇÃO DE ESTUDANTES DE ENFERMAGEM
}

\author{
John Victor dos Santos Silva ${ }^{1}$ \\ Ronildo Alves dos Santos ${ }^{2}$
}

\section{RESUMO}

A saúde mental é uma das áreas de atuação do enfermeiro e deve estar prevista na formação desses estudantes durante o curso de graduação. Este estudo tem como objetivo descrever as atividades práticas realizadas em dois Centros de Atenção Psicossocial, em 2017 e 2018, por estudantes do curso de Enfermagem de uma instituição de ensino superior de Alagoas. As práticas fazem parte do módulo de saúde mental de duas disciplinas ofertadas, uma no terceiro e a outra no quarto ano do curso. No delineamento deste relato, observam-se os aspectos que descrevem as atividades e campos práticos e mostra-se que a utilização desta alternativa como componente na formação dos futuros enfermeiros potencializa a construção do conhecimento sobre o campo da saúde mental. O ensino pautado no processo da reforma psiquiátrica, através de práticas em serviços substitutivos, como os Centros de Atenção Psicossocial, favorece o desenvolvimento de habilidades e competências na assistência da pessoa com transtornos mentais.

Palavras-chave: Serviços de saúde mental. Educação em Enfermagem. Educação superior.

\section{Como citar este documento - ABNT}

SILVA, John Victor dos Santos; SANTOS, Ronildo Alves dos. Atividades práticas em Centros de Atenção Psicossocial como estratégia na formação de estudantes de Enfermagem. Revista Docência do Ensino Superior, Belo Horizonte, v. 10, e020051, p. 1-16, 2020. DOI: https://doi.org/10.35699/22375864.2020.20051.

Recebido em: 22/04/2020 Aprovado em: $23 / 08 / 2020$ Publicado em: 09/10/2020

\footnotetext{
${ }^{1}$ Universidade de São Paulo (USP), Ribeirão Preto, SP, Brasil. ORCID ID: http://orcid.org/0000-0003-4671-102X. E-mail: johnvictor@usp.br 2 Universidade de São Paulo (USP), Ribeirão Preto, SP, Brasil. ORCID ID: http://orcid.org/0000-0003-3364-7727. E-mail: ronildo@eerp.usp.br
} 


\section{ACTIVIDADES PRÁCTICAS EN CENTROS DE ATENCIÓN PSICOSOCIAL COMO ESTRATEGIA EN LA FORMACIÓN DE ESTUDIANTES DE ENFERMERÍA}

\section{RESUMEN}

La salud mental es una de las áreas de especialización de la enfermera y se debe prever en la capacitación de estos estudiantes durante el curso de pregrado. Este estudio tiene como objetivo describir las actividades prácticas llevadas a cabo en dos Centros de Atención Psicosocial, en 2017 y 2018, por estudiantes de la carrera de Enfermería en una institución de educación superior en Alagoas. Las prácticas son parte del módulo de salud mental de dos asignaturas ofrecidas, en el tercer y el otro en el cuarto año del curso de pregrado. En el resumen de este informe, se observan los aspectos que describen las actividades y los campos prácticos y muestran que el uso de esta alternativa como componente en la capacitación de futuras enfermeras mejora la construcción de conocimiento sobre el campo de la salud mental. Enseñanza basada en el proceso de reforma psiquiátrica, a través de servicios sustitutos, como los Centros de Atención Psicosocial, favorece el desarrollo de habilidades y competencias en la atención y el trabajo con personas con trastornos mentales.

Palabras clave: Servicios de salud mental. Educación en Enfermaría. Educación superior.

\section{PRACTICAL ACTIVITIES AT PSYCHOSOCIAL CARE CENTERS AS A STRATEGY IN NURSING STUDENT TRAINING}

\section{ABSTRACT}

Mental health is among nurse's areas of expertise and students must be given the opportunity to learn about it during the undergraduate course. This study aims to describe the practical activities carried out at two Psychosocial Care Centers, in 2017 and 2018, by nursing course students at a higher education institution in Alagoas. The practices are part of the mental health module of two disciplines offered, one discipline in the 3rd and the other in the 4th year of the course. In the outline of this report are aspects that describe the activities and practical fields and show that the use of this alternative as a component in the training of future nurses enhances knowledge construction about the field of mental health. Teaching based on the psychiatric reform process, through practices in substitute services, such as Psychosocial Care Centers, favors the development of skills and competences in assisting people with mental disorders.

Keywords: Mental health services. Nursing education. Higher education. 


\section{INTRODUÇÃO}

O Centro de Atenção Psicossocial (CAPS) é um serviço de saúde mental que pertence ao Sistema Único de Saúde (SUS) e é direcionado para o cuidado e assistência de pessoas que possuem transtornos mentais. Caracterizado como um serviço territorial e de base comunitária, seu principal objetivo é ofertar o cuidado através do processo de reabilitação psicossocial, com a finalidade de proporcionar a reintegração familiar e social do indivíduo por meio de estratégias que tragam significado para sua existência, contribuam para o seu desenvolvimento pessoal e valorizem o viver em sociedade por meio do trabalho (BRASIL, 2017; ALVAREZ, 2019).

No Brasil, existem hoje sete modalidades diferentes de CAPS, cada um direcionado para diferentes públicos e territórios: CAPS I, II e III, destinados para adultos e idosos que possuem transtornos mentais severos e persistentes; o CAPS II infanto-juvenil, destinado ao tratamento de crianças e adolescentes que possuem transtornos mentais; e os CAPS ad II, III e IV, destinados para pessoas que fazem uso abusivo de álcool, crack e outras drogas. Todos eles seguem os princípios do SUS e estão dentro da Rede de Atenção Psicossocial, que interliga os CAPS aos demais dispositivos de saúde em todos os três níveis (BRASIL, 2017).

Para o funcionamento dos CAPS, além da estrutura física apropriada e materiais para realização do trabalho, é preciso recursos humanos de várias categorias profissionais que atuem tanto nas funções administrativas e operacionais quanto na assistência e no processo de reabilitação psicossocial dos usuários (BRASIL, 2002).

Assim, os profissionais de enfermagem fazem parte da equipe mínima de trabalho dos CAPS, em todas as suas modalidades. O enfermeiro, profissional de nível superior, torna-se responsável pela supervisão e gerenciamento das atividades das equipes de enfermagem dos CAPS, atuando, também, no trabalho interdisciplinar, na perspectiva do processo de reabilitação psicossocial dos usuários, juntamente com os outros profissionais da categoria de nível superior (SILVA; BRANDÃO, 2019).

Desta forma, as Diretrizes Nacionais Curriculares para a Enfermagem (DCN/ENF) orientam que os profissionais enfermeiros devem ser formados na perspectiva humanista e generalista, habilitado para atuar em todos os níveis de saúde e áreas do cuidado, assistindo o indivíduo, as famílias e comunidades nas suas necessidades em geral, incluindo as demandas provenientes da saúde mental (BRASIL, 2001).

O ensino da saúde mental é um componente obrigatório no currículo de Enfermagem, deve constar no projeto pedagógico do curso e proporcionar ao estudante uma aproximação com a área de atuação e o desenvolvimento de habilidades para o trabalho. Em geral, são ofertadas disciplinas ou módulos, com carga horária de atividades teórico-práticas e abordando 
conteúdos relacionados às políticas nacionais de saúde mental, assistência de enfermagem aos indivíduos com transtornos mentais e o trabalho interprofissional, proporcionando a construção do conhecimento sobre o campo da saúde mental, levando à preparação desses estudantes para o trabalho (TAVARES et al., 2016).

Cada instituição oferta as disciplinas de acordo com a realidade e perspectiva do campo em suas regiões. Assim, considera-se importante que, ao longo da formação, os alunos tenham a oportunidade de realizar práticas nos serviços de saúde mental, favorecendo a aproximação com o processo de trabalho e o desenvolvimento de competências de enfermagem para a saúde mental (SOUZA, 2016; VARGAS et al., 2018).

Embora muito importantes, as atividades práticas ou estágios no campo da saúde mental não são muito utilizados em alguns cursos de graduação em Enfermagem. Um estudo realizado por Vargas et al. (2018) mostrou um mapeamento das disciplinas de Psiquiatria e Saúde Mental nos cursos de graduação em Enfermagem no Brasil e inferiu que a grande maioria dessas disciplinas possui pouca ou nenhuma carga horária destinada à atividade prática, segundo suas ementas disciplinares, e que algumas instituições sequer ofertam a disciplina de Saúde Mental.

A não oferta de tal disciplina vai de encontro com as normativas das Diretrizes Curriculares Nacionais para a Enfermagem (DCN/ENF), que preconizam que o enfermeiro deve ser formado por meio de atividades teórico-práticas em todos os níveis de assistência à saúde e em todas as dimensões dos ciclos da vida, respondendo às demandas da sociedade nessa área e preparado para atuar em diversos serviços de saúde (BRASIL, 2001).

De acordo com as DCN/ENF, os cursos de graduação em Enfermagem devem ofertar os estágios curriculares supervisionados no último ano do curso de graduação, porém os alunos podem experienciar práticas profissionais ao longo do período do curso, o que poderá dar mais confiança e habilidades para a formação e para a atuação profissional (BRASIL, 2001). Entretanto, é comum que os estudantes não realizem estágios obrigatórios em serviços de saúde mental, pois o estágio obrigatório é, em geral, destinado aos cuidados na atenção básica e hospitalar, ficando apenas a critério das disciplinas de Saúde Mental realizar essas oportunidades de treinamento prático (SANTOS et al., 2016).

Desta forma, a motivação deste trabalho dá-se pela necessidade de compartilhar a vivência prática de estudantes de Enfermagem no campo da saúde mental, entendendo que as atividades práticas nos CAPS como componente da formação desses discentes podem favorecer uma maior assimilação dos conteúdos e da própria competência profissional nos serviços de saúde mental. 
Assim, o objetivo do presente trabalho é relatar a experiência das atividades práticas em Centros de Atenção Psicossocial como estratégia na formação de estudantes de Enfermagem para o trabalho no campo da saúde mental.

\section{DESCREVENDO A EXPERIÊNCIA}

Este trabalho é um relato de experiência sobre as atividades práticas realizadas nos CAPS por estudantes do curso de graduação em Enfermagem de uma instituição de ensino superior de Alagoas. A experiência aconteceu em 2017 no CAPS II, para pessoas com transtornos mentais severos e persistentes, através de uma disciplina anual ofertada para os estudantes do terceiro ano do curso de Enfermagem; e em 2018, no CAPS ad III, destinado para pessoas em uso abusivo de álcool, crack e outras drogas, através de uma disciplina anual ofertada para os alunos do quarto ano do curso da mesma instituição.

Essas atividades práticas são componentes do módulo de saúde mental, que é parte de duas disciplinas anuais. Os estudantes recebem o conteúdo teórico do módulo em sala de aula no mesmo período, porém as práticas acontecem ao longo do ano. Esses estudantes são divididos em grupos de no máximo cinco alunos para a realização de quatro atividades práticas nos CAPS, com quatro horas de duração cada uma. Os grupos não vão ao campo simultaneamente, sendo estabelecido um cronograma anual. Assim, o próximo grupo só vai ao campo quando o anterior termina suas atividades.

As atividades práticas têm como objetivo aproximar os estudantes dos serviços de saúde mental para que possam fazer o reconhecimento do trabalho que é realizado, aproximação com os profissionais e usuários, além de conhecer as estratégias para o trabalho e sua aplicação. Desta forma, as práticas foram estruturadas de forma que pudessem favorecer a visualização e aplicação do que é compartilhado em sala de aula.

\section{Centro de Atenção Psicossocial II: pessoas com transtornos mentais severos e persistentes}

As atividades práticas realizadas no CAPS II aconteceram no mês de junho de 2017, no turno da manhã, em dias de quarta-feira, acompanhadas pela professora responsável pelo módulo teórico e prático de Saúde Mental. Nessa modalidade de serviço, o foco é o tratamento e a reabilitação psicossocial de pessoas adultas com transtornos mentais severos e persistentes. As atividades foram organizadas segundo o cronograma apresentado na Figura 1: 


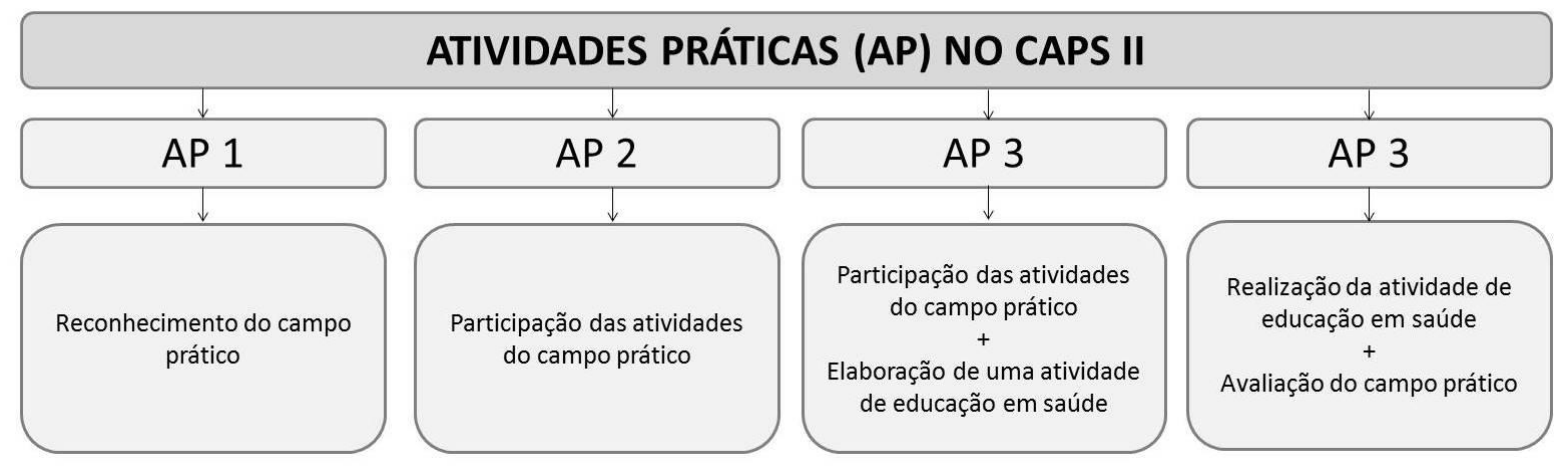

Figura 1 - Cronograma das atividades práticas no Centro e Atenção Psicossocial II para os estudantes do terceiro ano do curso de Enfermagem, em 2017.

Fonte: elaborado pelos autores, 2020.

A expectativa com o campo foi grande, haja vista que foi o primeiro momento e contato dos estudantes com serviços de saúde mental. No primeiro dia de prática, aconteceu o reconhecimento do serviço. Os alunos e a professora foram recebidos pela coordenadora do CAPS, que apresentou todo o ambiente, os profissionais e compartilhou um pouco sobre a rotina do serviço. Após sua apresentação, os alunos foram recebidos e acompanhados pela enfermeira do serviço, responsável por toda equipe e trabalho de enfermagem, para falar sobre a rotina dessa área no CAPS. Por se tratar do primeiro contato com o serviço, os alunos não participaram das atividades que eram trabalhadas no dia. O momento foi propício para conhecer o processo de trabalho do serviço e como suas ações são desenvolvidas.

No segundo dia de prática, os estudantes acompanharam as atividades e ações realizadas pela equipe de enfermagem no primeiro momento, como a verificação dos sinais vitais dos usuários, acompanhamento nas orientações e administrações de medicamentos. No segundo momento do dia, os estudantes acompanharam as oficinas terapêuticas realizadas pelos oficineiros na sala de artesanato, onde são realizados trabalhos de crochê, bordado, pintura, corte e costura, entre outros.

No terceiro dia de prática, em um primeiro momento, os estudantes acompanharam os profissionais do serviço numa atividade de sala de espera com todos os presentes sobre os cuidados com a higiene corporal. No segundo momento do dia, acompanharam o grupo terapêutico realizado pela terapeuta ocupacional e a psicóloga do serviço. A temática do grupo foi sobre as atividades diárias desenvolvidas em casa com os familiares. Ao final do dia, os alunos reuniram-se com a professora para elaborar uma atividade de educação em saúde a ser realizada com os usuários na semana seguinte. Após uma breve discussão, a temática escolhida pelos estudantes foi educação alimentar e atividade física. A proposta foi trabalhar com cartazes e imagens, também, e a realização de uma aula de zumba. 
No quarto e último dia de prática, os estudantes realizaram a atividade de educação em saúde no CAPS sobre alimentação saudável e atividade física. Foram elaborados dois cartazes, um com a frase "comer mais" e o outro com a frase "comer menos", e apresentados aos usuários imagens de vários tipos de alimentos. Foi explicada aos usuários a finalidade da atividade e pedido que eles colocassem no cartaz aquilo que eles deveriam comer mais e que deveriam comer menos. Ao final da atividade, os cartazes foram colados no mural do CAPS e feita uma exposição sobre algumas estratégias de alimentação saudável.

Após a atividade de educação em saúde, os alunos fizeram explicação sobre a importância da atividade física e ofertaram uma aula de zumba de trinta minutos para os usuários, familiares e profissionais do serviço. No término do dia, os estudantes reuniram-se com a professora para a avaliação do campo prático, na intenção de discutir sobre os aspectos positivos e o que deveria ser melhorado nas práticas. Foi entregue para cada aluno uma ficha avaliativa para discussão dos pontos positivos e negativos, assim como sugestões da rotina prática no CAPS.

Como aspectos positivos, os estudantes apontaram o acolhimento do CAPS ao recebê-los, a abertura dada pelos profissionais para que os discentes pudessem acompanhar as atividades e também a oportunidade de criar e aplicar uma atividade com os usuários. Como aspecto negativo, os estudantes pontuaram apenas a pouca disponibilidade de dias no campo prático.

\section{Centro de Atenção Psicossocial ad III: pessoas em uso abusivo de álcool e outras drogas}

As atividades práticas realizadas no CAPS ad III aconteceram em março de 2018, no turno da manhã, em dias de terça-feira, acompanhados pela professora responsável pelo módulo teórico e prático de saúde mental. Nessa modalidade de serviço, o foco é o tratamento e a reabilitação psicossocial de pessoas que fazem uso abusivo de álcool, crack e outras drogas.

Neste segundo campo, a expectativa era referente à realização de atividades de forma mais autônoma, permitindo o protagonismo dos estudantes. No primeiro dia de prática, aconteceu o reconhecimento do serviço, no qual os estudantes conheceram o ambiente e estrutura do CAPS, os profissionais, a rotina de trabalho, as atividades propostas, o funcionamento e os usuários do serviço. Desta vez, já no primeiro dia, os estudantes tiveram a oportunidade de participar das atividades que estavam sendo realizadas e, ao final do turno, realizaram os planejamentos para as próximas ações.

Diferentemente da estrutura das práticas da disciplina do ano anterior, a proposta desta vez é incentivar os estudantes a participarem e construírem as atividades e oficinas já no primeiro momento, como podemos observar no cronograma da Figura 2: 


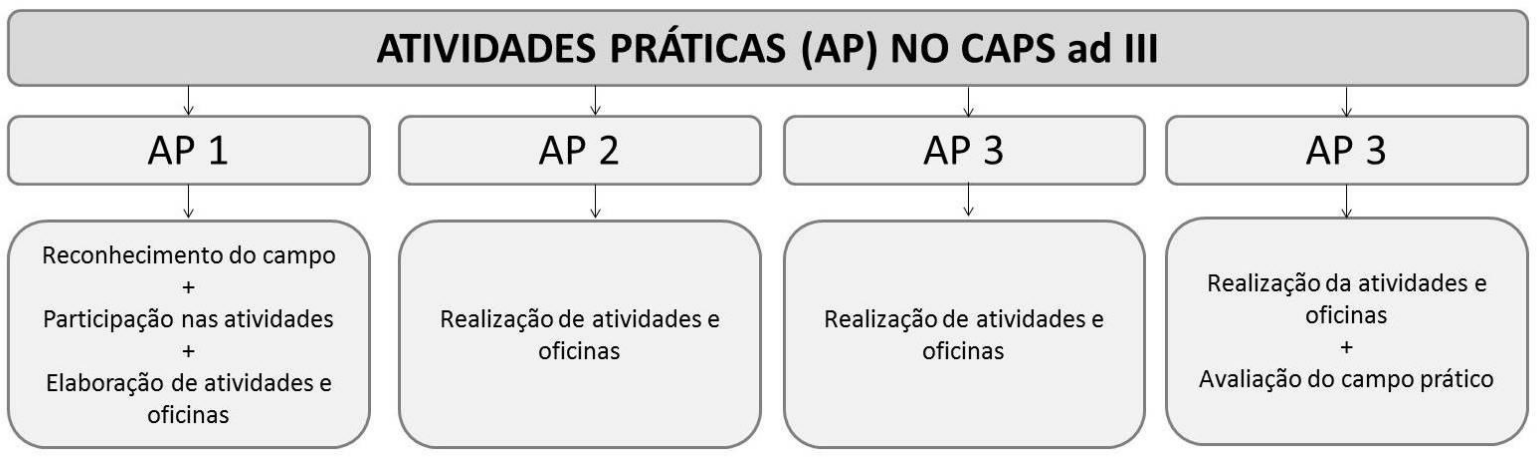

Figura 2 - Cronograma das atividades práticas no Centro e Atenção Psicossocial ad III para os estudantes do quarto ano do curso de Enfermagem, em 2018.

Fonte: elaborado pelos autores, 2020.

Para a decisão de quais atividades e oficinas seriam realizadas nas próximas práticas, os estudantes interagiram com os usuários, para conhecer mais a história de cada um e planejar quais estratégias seriam utilizadas. Ao final deste momento, os estudantes reuniram os usuários e compartilharam quais opções de atividades poderiam ser realizadas, conhecendo suas opiniões sobre o que gostariam de fazer nas próximas semanas. Assim, as opções que surgiram foram: jogos em geral, cinema e desenho, com foco nas relações interpessoais, a colaboração e o trabalho em equipe.

No segundo dia de prática, os estudantes de Enfermagem realizaram uma sessão de "Cinema no CAPS" com os usuários, na qual foram apresentados dois filmes de curta metragem: uma animação sobre o trabalho em equipe ${ }^{3}$; e um outro sobre a família e as nossas relações interpessoais ${ }^{4}$. Ao final do primeiro filme, os estudantes propuseram uma discussão com os usuários sobre as atividades colaborativas e o trabalho em equipe. Logo mais, ao final do segundo filme, os estudantes levantaram questões sobre as relações familiares. $O$ foco foi mostrar aos usuários que o CAPS é um ambiente propício para fortalecimento das relações, seja usuário-usuário e usuário-profissional.

Essas discussões foram possíveis porque, em geral, os usuários dos CAPS ad são pessoas que possuem o nível de consciência, comunicação e colaboração preservado para manter, diferentemente daqueles do outro CAPS, que possuem essas funções mais comprometidas, impossibilitando, em alguns momentos, o estabelecimento das relações interpessoais.

No terceiro dia de prática, os estudantes dividiram-se e fizeram, cada um, pequenos grupos com os usuários e realizaram jogos como dominó, jogos de cartas, dinâmicas com bolas, entre outros. A intenção dos jogos era trazer novamente à tona a perspectiva das relações com os

\footnotetext{
${ }^{3}$ PRESTO: Digiotagione \& his hat of wonder - Walt Disney and Pixar Animation Studios Cartoon.

${ }^{4}$ La Luna - Pixar Animation Studios Cartoon.
} 
demais, seguindo na linha proposta da atividade anterior. Ao final dos jogos, um profissional do CAPS realizou uma atividade de dança, na qual os estudantes também participaram e incentivaram a participação de todos.

Na quarta e última semana, os estudantes fizeram uma oficina de corte, colagem e desenhos livres. Desta vez, foi reforçando o trabalho em equipe, a colaboração e a importância das relações saudáveis. Ao passo que os usuários realizavam suas pinturas e desenhos, era enfatizada a importância de ajudar o outro e de compartilhar os materiais.

Ao final do dia, os estudantes reuniram-se com a professora e fizeram a avaliação do módulo prático na ficha avaliativa, expondo, mais uma vez, os pontos positivos e negativos, suas percepções sobre o aprendizado e o trabalho, tanto para melhoria da disciplina quanto para o seu desenvolvimento como futuros enfermeiros.

Positivamente foram pontuados os seguintes aspectos: maior abertura para o trabalho e ações desenvolvidas e conduzidas pelos próprios estudantes, das quais puderam ser protagonistas (diferentemente da experiência anterior, na qual ficavam como auxiliadores da atividade), e também maior confiança naquilo que foi realizado. Como aspecto negativo, os estudantes pontuaram novamente sobre a pouca carga horária para as atividades práticas, além de não terem sido oportunizados momentos para realização da consulta de enfermagem, um dos importantes instrumentos do processo de trabalho nessa área e que permite a realização de procedimentos e avaliações, como o exame físico e mental, para conhecer mais sobre a situação clínica do usuário.

\section{DISCUSSÃO}

No delineamento deste relato, pode-se verificar que os estudantes de Enfermagem de uma instituição de ensino superior pública tiveram a oportunidade de vivenciar o trabalho no campo da saúde mental, através das práticas realizadas em dois Centros de Atenção Psicossocial. Esses momentos foram importantes para que os estudantes pudessem visualizar na prática o que havia sido abordado em sala de aula, como a realização de ações de educação em saúde, atividades recreativas e oficinas.

A maior contribuição que as atividades práticas nos CAPS puderam oferecer aos estudantes foi justamente a oportunidade de colocar em prática o conhecimento que foi construído e as ferramentas profissionais que foram desenvolvidas ao longo dos conteúdos teóricos discutidos em sala de aula. Aprende-se melhor quando se oportuniza momentos para fazer, executar, realizar. $E$ foi justamente isso que vivenciaram os estudantes de Enfermagem nesses dois campos práticos. 
Um estudo realizado por Oliveira et al. (2018), através do método de simulação prática, mostrou que os alunos têm mais facilidade de assimilação dos conteúdos teóricos sobre a assistência em saúde mental quando são oportunizados momentos para o desenvolvimento prático, pois permite que o processo de ensino-aprendizagem extrapole os muros da sala de aula e sejam colocados em prática, como numa situação real de assistência na saúde mental.

Integrar os conteúdos teóricos com experiências práticas, principalmente nos próprios serviços de saúde mental, como é o exemplo do CAPS, torna-se um fator potencializador na formação de futuros profissionais capacitados para atuar nos serviços de saúde mental de forma mais qualificada (MENDES et al., 2018).

Por isso, essas atividades práticas realizadas no Centro de Atenção Psicossocial configuram-se como um dos recursos utilizados pelos docentes das disciplinas da saúde mental como oportunidade para desenvolver competências e habilidades de enfermagem para a assistência em saúde das pessoas com transtornos mentais e que necessitam, principalmente, de uma boa relação entre profissional-usuário (TAVARES et al., 2016).

É sabido que a vivência no campo prático possibilita uma melhor aproximação com a realidade da assistência em saúde, através dos seus profissionais, usuários e rotina do serviço. Essa perspectiva de trabalho através dos seus atores nos serviços possibilita que os estudantes conheçam as estratégicas e reflitam sobre as diferentes formas de trabalho (MARRAN; SILVA; SALES, 2017).

Embora existam outros serviços, o CAPS ainda é considerado o principal serviço de saúde mental no território brasileiro, tornando-se, para os alunos de Enfermagem, a principal oportunidade de trabalho na assistência às pessoas com transtornos mentais. Desta forma, quando o aluno tem a oportunidade de vivenciar a rotina do serviço e também participar das atividades realizadas, isso faz com que ele esteja mais preparado para atuar futuramente no trabalho como enfermeiro, em qualquer modalidade de CAPS (REIS; PEDRAS, 2017).

Para isso, é importante que as instituições de ensino firmem parcerias com as secretarias de saúde para que possam ser utilizados os CAPS como campo de prática para os estudantes. Ainda assim, a utilização do CAPS como campo prático vai depender, também, de como é organizada a Rede de Atenção Psicossocial na região e como estão distribuídos esses serviços nos territórios, e a presença ou não deles.

Pode-se perceber a evolução da proposta das práticas entre os dois momentos distintos. Enquanto que no primeiro momento no CAPS II os estudantes estiveram mais como participantes das atividades já existentes no serviço e só ao final puderam realizar uma ação planejada por eles, no CAPS ad III já é possível visualizar esses estudantes como protagonistas no planejamento e execução das ações nos serviços. 
Essa evolução do estudante entre os campos práticos dá-se, principalmente, quando a organização das disciplinas favorece diferentes momentos para o amadurecimento profissional e realiza o acompanhamento dos estudantes desde o início de seu processo de formação em determinada área e campo. Por isso, torna-se importante que os professores atuem como facilitadores desse processo, colocando os estudantes em um papel mais ativo no seu próprio processo de formação.

Sabe-se que o campo da saúde mental exige, principalmente, a prática da interdisciplinaridade e interprofissionalidade. No âmbito da formação dos estudantes de saúde, Silva e Ribeiro (2018) apontam que muitas vezes só é possível visualizar a sua aplicação nos ambientes de prática e estágios, haja vista que na sala de aula as turmas são sempre específicas de cada curso, não tendo muita interação com os demais. Por isso, como proposta do segundo campo prático, os estudantes planejaram e realizaram atividades com os usuários contando, também, com a participação dos demais profissionais do serviço, principalmente com a equipe de enfermagem.

Essa aproximação com os profissionais de Enfermagem dos CAPS também proporciona a visualização efetiva do papel do enfermeiro nos serviços. Um estudo realizado por Silva e Brandão (2019) mostra que a enfermagem ainda não tem grande destaque nas ações de reabilitação psicossocial realizadas pelas equipes multiprofissionais, restringindo-se mais aos papeis burocráticos ou na assistência de enfermagem, como administração e orientações sobre medicamentos, pequenos curativos, entre outros.

A respeito das atividades que foram realizadas pelos alunos, é importante salientar que as oficinas e atividades, para serem consideradas terapêuticas, precisam ter a finalidade de promover transformação nos indivíduos, seja uma transformação na percepção de mundo, nas atitudes, nas condutas, entre outros, assim como os estudantes puderam construir atividades distintas, mas com a finalidade de propor a reflexão e o exercício das relações em grupo e colaboração (CONSTANTINIDIS et al., 2018).

De toda forma, as atividades realizadas pelos estudantes nos CAPS são as ações mais desenvolvidas nos serviços, como o acompanhamento das atividades realizadas pelos usuários, oficinas e atividades terapêuticas, o acolhimento, a conversa terapêutica, educação em saúde, entre outras. Algumas atividades são realizadas especificamente pela equipe de enfermagem, mas em sua maioria, as ações e grupos são realizados de forma coletiva e interprofissional (SILVA; BRANDÃO; OLIVEIRA, 2019).

Essa reflexão sobre quais atividades os estudantes poderiam utilizar só seria possível através de momento de observação e também de comunicação entre os alunos e os usuários. Enquanto no primeiro campo prático a escolha pela temática se deu mais pela observação e alguns diálogos com os usuários, no segundo campo os estudantes puderam estabelecer uma 
comunicação direta com todos, a fim de extrair deles as suas necessidades, o que é de muita importância no desenvolvimento do processo de reabilitação psicossocial.

Essa comunicação com os usuários deve acontecer respeitando as limitações de todos. Enquanto no CAPS II a comunicação é mais limitada pelas condições severas e persistentes dos indivíduos, sendo mais curta, no CAPS ad III é possível estabelecer uma comunicação mais duradoura, pois os usuários são mais colaborativos e conscientes do acontecimento (JALLES; SANTOS; REINALDO, 2017).

Nesse processo de formação dos estudantes de Enfermagem, conhecer a realidade dos serviços como os CAPS favorece uma formação mais pautada na perspectiva da reforma psiquiátrica, em que o indivíduo que possui transtornos mentais pode ser tratado dentro do cenário da comunidade e trabalhar em prol da sua inserção social (MARTINS et al., 2018).

Assim, os objetivos propostos pelas duas disciplinas, em seus dois campos práticos, apresentados nas Figuras 1 e 2, foram alcançados. Os estudantes fizeram o reconhecimento dos serviços, conheceram os profissionais e o processo de trabalho, participaram da realização dos grupos terapêuticos, realizaram uma ação de educação em saúde, atividades educativas, exercitaram a comunicação terapêutica e a escuta qualificada, atuaram de forma interprofissional e interdisciplinar e realizaram a autoavaliação e a avaliação da disciplina.

Desta forma, as atividades práticas proporcionaram aos estudantes uma formação ampliada, contribuindo para a formação do enfermeiro generalista, preparando-o para trabalhar em todos os campos em que a enfermagem atua, incluindo a saúde mental, através dos CAPS, como acontece em maternidades, hospitais, unidades de saúde, entre outros serviços. Assim, quando o estudante de Enfermagem é inserido num serviço de saúde mental, ele tem a oportunidade de ter uma formação mais qualificada e completa para atuar na assistência das pessoas com transtornos mentais.

Assim, levando em consideração a responsabilidade social das instituições de ensino superior em formar profissionais qualificados para a assistência em saúde, quanto mais elas oportunizarem atividades práticas ao longo do processo de formação na graduação em Enfermagem, principalmente nos próprios serviços de saúde, mais preparados os estudantes estarão para o cuidado integral em todos os níveis de saúde.

\section{CONSIDERAÇÕES FINAIS}

No delineamento deste relato, observam-se os aspectos que descrevem as atividades e campos práticos e mostra-se que a utilização desta alternativa como componente na formação dos futuros enfermeiros potencializa a construção do conhecimento sobre o campo da saúde mental, pautada no processo da reforma psiquiátrica, através dos serviços 
substitutivos, como o CAPS, e também no desenvolvimento das habilidades e competências na assistência e no trabalho junto à pessoa com transtornos mentais.

Mostra-se que foi possível aplicar algumas atividades, desenvolvidas pelos estudantes, com os conteúdos adquiridos em sala de aula e com recursos dos próprios serviços. Assim, a realização das oficinas e atividades, como a educação em saúde, reforça a necessidade de preparação e trabalho para a sua realização.

Desta forma, os campos práticos mostram-se de grande valor no processo de formação dos estudantes, sabendo que os profissionais enfermeiros fazem parte dos recursos humanos necessários para a assistência das pessoas com transtornos mentais em todos os níveis de saúde, principalmente nos CAPS.

Salienta-se que essa experiência mostra um recorte temporal e regional, no qual foram descritas as atividades realizadas por estudantes de uma única instituição, sendo necessárias outras investigações para se conhecer como as demais instituições vêm desenvolvendo as atividades práticas com os estudantes de Enfermagem e quais serviços são percorridos.

\section{REFERÊNCIAS}

ALVAREZ, Patricia Elizabeth Sanz de. Percepciones y expectativas que inciden en la vinculación de los usuarios a los Centros de Atención Psicosocial en Brasil. Interface (Botucatu), Botucatu, v. 23, p. 1-15, 2019. Disponível em:

https://www.scielo.br/pdf/icse/v23/1807-5762-icse-23-e190117.pdf. Acesso em: 15 jun. 2020. DOI: http://dx.doi.org/10.1590/interface.190117.

BRASIL, CÂMARA NACIONAL DE EDUCAÇÃO. Resolução CNE/CES no 3. Institui Diretrizes Curriculares Nacionais do Curso de Graduação em Enfermagem. Câmara de Educação Superior. Brasília, DF. 7 nov. 2001. Disponível em:

http://portal.mec.gov.br/cne/arquivos/pdf/CES03.pdf. Acesso em: 8 abr. 2020.

BRASIL, MINISTÉRIO DA SAÚDE. Portaria no 336. Institui os Centros de Atenção Psicossocial e demais providências. Brasília, DF. 19 fev. 2002. Disponível em:

http://bvsms.saude.gov.br/bvs/saudelegis/gm/2002/prt0336_19_02_2002.html. Acesso em: 8 abr. 2020.

BRASIL, MINISTÉRIO DA SAÚDE. Portaria no 3588. Altera as Portarias de Consolidação no 3o e no 6, de 28 de setembro de 2017, para dispor sobre a Rede de Atenção Psicossocial, e dá outras providências, 2017. Brasília, DF. 21 dez. 2017. Disponível em:

http://bvsms.saude.gov.br/bvs/saudelegis/gm/2017/prt3588_22_12_2017.html. Acesso em: 8 abr. 2020.

CONSTANTINIDIS, Terezinha Cid et al. Concepções de profissionais de saúde mental acerca de atividades terapêuticas em CAPS. Trends in Psychology, Ribeirão Preto, v. 26, n. 2, p. $911-$ 
926, 2018. Disponível em: http://www.scielo.br/pdf/tpsy/v26n2/2358-1883-tpsy-26-020911.pdf. Acesso em: 13 abr. 2020. DOI: https://doi.org/10.9788/tp2018.2-14pt.

JALLES, Marina Paranhos; SANTOS, Viviane Silva Januário dos; REINALDO, Amanda Márcia dos Santos. Análise da produção científica sobre comunicação terapêutica no campo da saúde, saúde mental e álcool e outras drogas. Revista de Medicina, Ribeirão Preto, v. 96, n. 4, p. 232-240, 2017. Disponível em:

https://www.revistas.usp.br/revistadc/article/view/122431. Acesso em: 15 jun. 2020. DOI: https://doi.org/10.11606/issn.1679-9836.v96i4p232-240.

MARRAN, Ana Lúcia; SILVA, Mariana Moreira da; SALES, Cibele de Moura. Potencialidades e desafios de uma estratégia para aulas práticas onde a teoria pode vir depois. Laplage em Revista, Sorocaba, v. 3, n. 2, p. 195-205, 2017. Disponível em:

https://www.redalyc.org/jatsRepo/5527/552756522017/552756522017.pdf. Acesso em: 15 jun. 2020. DOI: https://doi.org/10.24115/S2446-6220201732328p.195-205.

MARTINS, Gizele da Conceição Soares et al. Ensino de graduação em Enfermagem em Saúde Mental como aliado à consolidação do movimento de Reforma Psiquiátrica. Escola Anna Nery, Rio de Janeiro, v. 22, n. 4, 2018. Disponível em:

http://www.scielo.br/pdf/ean/v22n4/pt_1414-8145-ean-22-04-e20180164.pdf. Acesso em: 13 abr. 2020. DOI: https://doi.org/10.1590/2177-9465-ean-2018-0164.

MENDES, Aida Cruz et al. Educação em enfermagem de saúde mental e psiquiatria no curso de licenciatura em Enfermagem. SMAD Revista Eletrônica Saúde Mental Álcool e Drogas, Ribeirão Preto, v. 14, n. 2, p. 73-83, 2018. Disponível em: http://www.periodicos.usp.br/smad/article/view/155623. Acesso em: 15 jun. 2020. DOI: 10.11606/issn.1806-6976.smad.2018.000366.

OLIVEIRA, Saionara Nunes de et al. Da teoria à prática, operacionalizando a simulação clínica no ensino de Enfermagem. Revista Brasileira de Enfermagem, Brasília, v. 71, p. 17911798, 2018. Disponível: http://www.scielo.br/scielo.php?script=sci_arttext\&pid=S003471672018001001791\&lng=en\&nrm=iso. Acesso em: 15 jun. 2020. DOI: https://doi.org/10.1590/0034-7167-2017-0180.

REIS, Alex G.; PEDRAS, Evelin Regina de Souza. Percepção de discentes de Enfermagem de uma instituição de ensino superior sobre o ensino de saúde mental. Revista Brasileira de Ciências da Vida, Sete Lagoas, v. 5, n. 4, p. 21-21, 2017. Disponível em:

http://jornal.faculdadecienciasdavida.com.br/index.php/RBCV/article/view/439/214. Acesso em: 13 abr. 2020.

SANTOS, Josenaide Egracia dos et al. Processos formativos da docência em saúde mental nas graduações de Enfermagem e Medicina. Revista Portuguesa de Enfermagem de Saúde Mental, Lisboa, n. SPE4, p. 85-92, 2016. Disponível em: http://www.scielo.mec.pt/pdf/rpesm/nspe4/nspe4a13.pdf. Acesso em: 13 abr. 2020. DOI: http://dx.doi.org/10.19131/rpesm.0146. 
SILVA, John Victor dos Santos; BRANDÃO, Thyara Maia. A enfermagem dos Centros de Atenção Psicossocial de uma capital do Nordeste do Brasil. Revista de Enfermagem e Atenção à Saúde, Uberaba, v. 8, n. 1, 2019. Disponível em:

http://seer.uftm.edu.br/revistaeletronica/index.php/enfer/article/view/3379. Acesso em: 15 jun. 2020. DOI: https://doi.org/10.18554/reas.v8i1.3379.

SILVA, John Victor dos Santos; BRANDÃO, Thyara Maia; OLIVEIRA, Keila Cristina Pereira do Nascimento. Ações e atividades desenvolvidas pela enfermagem no Centro de Atenção Psicossocial: revisão integrativa. Revista de Enfermagem e Atenção à Saúde, Uberaba, v. 7, n. 3, p. 137-149, 2019. Disponível em:

http://seer.uftm.edu.br/revistaeletronica/index.php/enfer/article/view/3115. Acesso em: 15 jun. 2020. DOI: https://doi.org/10.18554/reas.v7i2.3115.

SILVA, John Victor dos Santos; RIBEIRO, Mara Cristina. O docente de Enfermagem e sua percepção sobre as ações integrativas na Saúde e na formação interprofissional. Revista Docência do Ensino Superior, v. 8, n. 2, p. 245-261, 2018. Disponível em:

https://periodicos.ufmg.br/index.php/rdes/article/view/2464. Acesso em: 15 jun. 2020. DOI: https://doi.org/10.35699/2237-5864.2018.2464.

SOUZA, Maria Conceição Bernardo de Mello. O ensino de Enfermagem Psiquiátrica/Saúde Mental: avanços, limites e desafios. SMAD Revista Eletrônica Saúde Mental Álcool e Drogas, Ribeirão Preto, v. 12, n. 3, p. 139-146, 2016. Disponível em:

https://www.revistas.usp.br/smad/article/view/120777/117844. Acesso em: 8 abr. 2020. DOI: https://doi.org/10.11606/issn.1806-6976.v12i3p139-146.

TAVARES, Cláudia Mara de Melo et al. Competências específicas do enfermeiro de saúde mental enfatizadas no ensino de graduação em Enfermagem. Revista Portuguesa de Enfermagem de Saúde Mental, Porto, n. spe4, p. 25-32, 2016. Disponível em:

http://www.scielo.mec.pt/scielo.php?script=sci_arttext\&pid=S1647-

21602016000400004\&lng=pt\&nrm=iso. Acesso em: 15 jun. 2020. DOI:

http://dx.doi.org/10.19131/rpesm.0137.

VARGAS, Divane de et al. O ensino de enfermagem psiquiátrica e saúde mental no Brasil: análise curricular da graduação. Texto \& Contexto Enfermagem, Florianópolis, v. 27, n. 2, 2018. Disponível em: http://www.scielo.br/scielo.php?script=sci_arttext\&pid=S010407072018000200316\&lng=pt\&nrm=iso. Acesso em: 8 abr. 2020 . DOI: http://dx.doi.org/10.1590/0104-070720180002610016. 
Atividades práticas em Centros de Atenção Psicossocial como estratégia na formação de estudantes de Enfermagem

John Victor dos Santos Silva, Ronildo Alves dos Santos

\section{John Victor dos Santos Silva}

Enfermeiro. Mestrando em Enfermagem Psiquiátrica pela Escola de Enfermagem de Ribeirão Preto da Universidade de São Paulo (EERP/USP). Integrante do Grupo de pesquisa "Ensino, Saúde e Sociedade" da Universidade Estadual de Ciências da Saúde de Alagoas (UNCISAL), vinculado ao CNPq.

john.setedejulho@gmail.com

\section{Ronildo Alves dos Santos}

Doutor. Professor da Escola de Enfermagem de Ribeirão Preto (EERP/USP) no departamento de Enfermagem Psiquiátrica e Ciências Humanas. Orientador no Programa de Pós-graduação em Enfermagem Psiquiátrica.

ronildo@eerp.usp.br 